\title{
Investigation of Transparency of FWM in SOA to Advanced Modulation Formats Involving Intensity, Phase, and Polarization Multiplexing
}

\author{
Giampiero Contestabile, Luca Banchi, Student Member, IEEE, Marco Presi, and Ernesto Ciaramella, Member, IEEE
}

\begin{abstract}
We experimentally investigate the real transparency of four-wave mixing (FWM) in semiconductor optical amplifiers to modulation formats involving intensity, phase, and polarization multiplexing. We exploit two different FWM polarization-independent schemes (that make use of two pumps) to wavelength-convert $40 \mathrm{~Gb} / \mathrm{s}$ single-polarization and $80 \mathrm{~Gb} / \mathrm{s}$ polarization-multiplexed signals in case of both nonreturn-to-zero $O N-O F F$ keying (NRZ-OOK) and NRZ differential phase-shift keying modulation formats. We found that, although FWM conversion is transparent to modulation formats employing phase and intensity, polarization-multiplexed signals pose serious limitations to all-optical processing transparency.
\end{abstract}

Index Terms-Four-wave mixing (FWM), semiconductor optical amplifier (SOA), wavelength conversion.

\section{INTRODUCTION}

$\mathbf{F}$ UTURE optical networks should take advantage from optical transparency, i.e., a number of switching functions performed directly in the optical domain in order to reduce the number of power-hungry high-speed optical-electronic-optical conversions at network nodes. Nowadays, on the other hand, the clear trend in order to increase the bit rate of optical channels is the use of advanced modulation formats that exploit a mix of intensity and phase modulation often combined with polarization multiplexing (PolMux) [1]. As an example, PolMux differential quaternary phase-shift keying (DQPSK) is one of the most promising modulation formats for future $100 \mathrm{~Gb} / \mathrm{s}$ systems [2]. However, these two technological trends lead to a clear technical conflict. Indeed, while fully transparent all-optical processing for advanced modulation formats would give the largest power and cost convenience (reducing the overall number of complex transmitter and receiver pairs in the networks), their practical implementation could result in a very hard task.

As an example, the wavelength conversion of complex signals involving intensity and phase can only be realized using optical coherent techniques that are inherently polarization dependent [3]. In practice, this limits the choice to a few techniques (i.e., nonlinear coherent mixing in semiconductors, fibers, and periodically poled $\mathrm{LiNbO}_{3}$ ) that must be implemented in modified

Manuscript received November 14, 2008; revised February 27, 2009. First published May 15, 2009; current version published August 26, 2009. This work was supported in part by Ericsson SpA under a grant.

The authors are with the Scuola Superiore Sant'Anna, University of Pisa, 56124 Pisa, Italy (e-mail: contesta@sssup.it).

Color versions of one or more of the figures in this paper are available online at http://ieeexplore.iee.org.

Digital Object Identifier 10.1109/JLT.2009.2023091

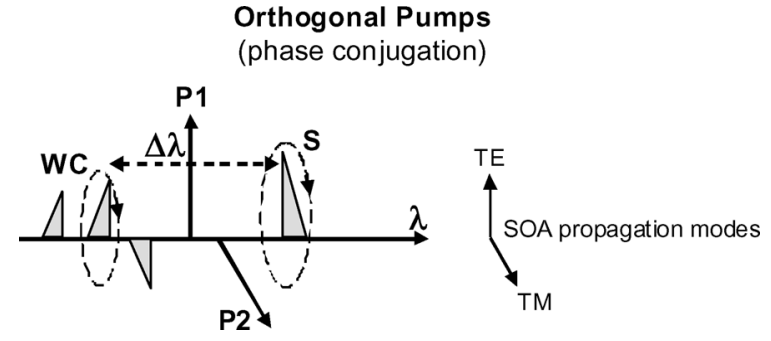

Fig. 1. Schematic of the orthogonal pumps' FWM configuration.

configurations to make them polarization independent [4], [5]. Among them, four-wave mixing (FWM) in semiconductor optical amplifiers (SOAs) is particularly promising as it uses an active nonlinear medium. Hence, it works at low input power, with good conversion efficiency, and has the advantage of potential photonic integration. Moreover, despite its intrinsic polarization dependence, two modified schemes employing two pumps can make FWM polarization independent to a good extent [4], [6]. In this paper, we experimentally study the real transparency of FWM in SOAs to $80(2 \times 40) \mathrm{Gb} / \mathrm{s}$ PolMux signals in case of both nonreturn-to-zero $O N-O F F$ keying (NRZ-OOK) and NRZ differential phase-shift keying (DPSK) modulation formats. We exploit two schemes to obtain polarization independence in an SOA: FWM with two orthogonal [6] or parallel [4] pumps. Results show that despite transparency to intensity and phase that can be obtained, there are critical issues for full optical transparence to PolMux signals.

The paper is organized as follows. In Section II, we describe the orthogonal pumps scheme and report the wavelength conversion results. In Section III, we introduce the scheme with parallel pumps and relative results. Finally, in Section IV, we compare and discuss the results.

\section{FWM WITH ORTHOGONAL PUMPS}

The scheme of FWM conversion with orthogonal pumps is sketched in Fig. 1. Assuming to use an SOA that is polarization independent, in case of orthogonal pumps, the two pumps P1 and $\mathrm{P} 2$ are aligned to the TE and TM axes of the device. A signal $\mathrm{S}$ having random polarization has components along both axes; hence, it beats with both pumps.

Therefore, in this scheme, there are three FWM copies [6] (that are phase conjugate as in usual single-pump configuration). Among these, the central one, resulting from the double beating with both pumps, has two components along the two 


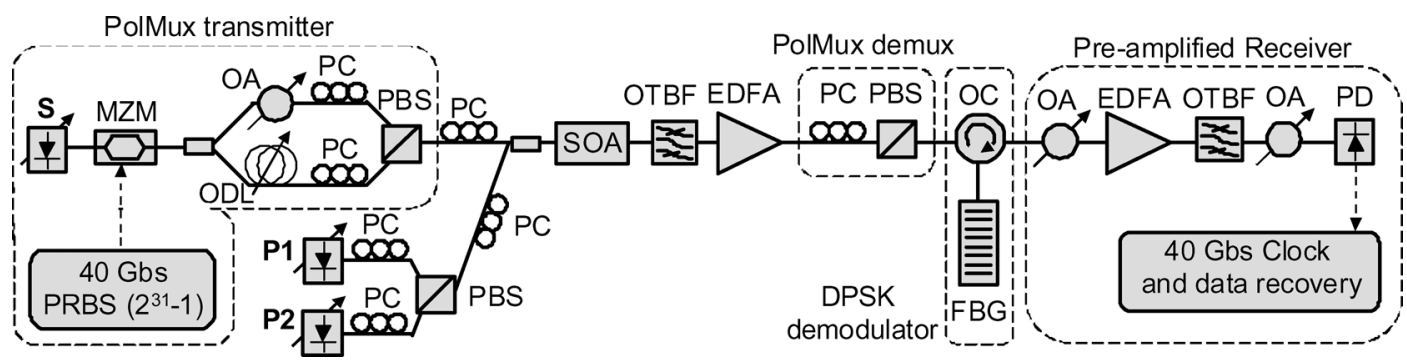

Fig. 2. Experimental setup. OA: optical attenuator; PC: polarization controller; ODL: optical delay line; OC: optical circulator; FBG: fiber Bragg grating; OTBF: optical tunable bandpass filter.

axes. If these two components have the same conversion efficiency (i.e., the pumps have the same power and are very close to each other with respect to conversion detuning), the resulting converted signal is polarization independent [6]. To test this scheme, we used the experimental setup reported in Fig. 2. We used an $80 \mathrm{~Gb} / \mathrm{s}$ tunable PolMux transmitter. It was made of a $40 \mathrm{~Gb} / \mathrm{s}$ patter generator (using $2^{31}-1$ pseudorandom bit sequences), a tunable continuous-wave $(\mathrm{CW})$ laser with around $10 \mathrm{MHz}$ linewidth, and a $40 \mathrm{~Gb} / \mathrm{s} \mathrm{LiNbO}_{3}$ Mach-Zehnder modulator to generate (changing the bias point and the data voltage) both NRZ-OOK and NRZ-DPSK data. A $3 \mathrm{~dB}$ splitter and an optical delay line ( $250 \mathrm{~m}$ single-mode fiber) were used to phase decorrelate the two signals that were polarization controlled, and then, multiplexed by means of a polarization beam splitter (PBS)/combiner with a $17 \mathrm{~dB}$ extinction ratio (ER). Two other tunable laser sources were used to generate the pumps P1 and P2. They were polarization controlled, and then, coupled by another PBS. The SOA (CIP XN OEC 1550) was a highly nonlinear multiquantum-well device with about $33 \mathrm{~dB}$ small-signal gain, around $15 \mathrm{dBm}$ output saturation power, and a polarization-dependent gain (PDG) of $1 \mathrm{~dB}$ when biased at $550 \mathrm{~mA}$. The FWM signals were selected by a flat top tunable filter of around $0.4 \mathrm{~nm}$ bandwidth and more than $40 \mathrm{~dB}$ isolation and amplified by a low-noise erbium-doped fiber amplifier (EDFA). Polarization demultiplexing was manually performed by a polarization controller and another PBS with $17 \mathrm{~dB}$ ER. In the DPSK case, the demodulation was performed by means of optical sharp Gaussian filtering (bandwidth: $26 \mathrm{GHz}$ as demonstrated in [7]) using a circulator together with a periodic fiber Bragg grating filter. We used a preamplified receiver composed of an EDFA, an optical bandpass filter, a $40 \mathrm{~Gb} / \mathrm{s}$ error detector, and a clock and data recovery.

In order to properly set the orthogonal pumps configuration, we aligned the polarization of $\mathrm{P} 1$ and $\mathrm{P} 2$ to the two SOA propagation axes. This was made by minimizing the FWM product between them. Indeed, despite the orthogonal condition set by the PBS, the SOA-induced birefringence causes pumps' FWM if they are not exactly set on the axes.

We performed a study of the conversion efficiency and output optical SNR (OSNR) as a function of the input signal power and detuning. We chose the optimal values that were compatible with the other assumptions requested by the scheme to keep the conversion detuning reasonable larger than the detuning between the pumps. The data regarding all the involved input signals and the wavelength-converted (WC) ones (wavelength detuning, conversion efficiency, OSNR, and residual polarization
TABLE I

EXPERIMENTAL POWER LEVELS

\begin{tabular}{|c|c|c|c|c|}
\hline & P1 & P2 & S & WC \\
\hline$\lambda(\mathbf{n m})$ & 1559.3 & 1560.1 & 1561.8 & 1557.6 \\
\hline $\begin{array}{c}\text { OOK } \\
\text { Power } \\
\text { (dBm) }\end{array}$ & 9 & 8 & 1 & -9 \\
\hline $\begin{array}{c}\text { DPSK } \\
\text { Power } \\
(\mathbf{d B m})\end{array}$ & 9 & 8 & 2 & -8.5 \\
\hline
\end{tabular}

TABLE II

WAVELENGTH CONVERSION RESULTS

\begin{tabular}{|c|c|c|}
\cline { 2 - 3 } \multicolumn{1}{c|}{} & OOK & \multicolumn{1}{c|}{ DPSK } \\
\hline$\Delta \lambda(\mathbf{n m})$ & 4.2 & 4.2 \\
\hline $\begin{array}{c}\text { Conversion } \\
\text { Efficiency } \\
(\mathbf{d B})\end{array}$ & -10 & -10.5 \\
\hline $\begin{array}{c}\text { OSNR } \\
(\mathbf{d B} / \mathbf{0 . 1 n m})\end{array}$ & 32 & 33 \\
\hline $\begin{array}{c}\text { Residual } \\
\text { pol. depen. } \\
\text { (dB) }\end{array}$ & 1.1 & 0.9 \\
\hline
\end{tabular}

dependence) are summarized in Tables I and II for OOK and DPSK, respectively. In the case of single polarization, we modified the setup opening one arm of the PolMux transmitter and adjusted the $\mathrm{S}$ input power to the same value as used for the PolMux case.

The typical optical spectra at the SOA output and the BER versus power (at the preamplifier) for the single polarization and PolMux cases (40 and $80 \mathrm{~Gb} / \mathrm{s}$, respectively) are reported in Fig. 3 for the OOK format and in Fig. 4 for the DPSK format. Corresponding eye diagrams are also reported in the insets. We called Ch.1 and Ch.2 the two PolMux channels. The conversion detuning $(\Delta \lambda)$ is $4.2 \mathrm{~nm}$, the conversion efficiency is around $-10 \mathrm{~dB}$, and the residual polarization dependence is around $1 \mathrm{~dB}$ for both cases (residual polarization dependence taken recording the maximum and minimum power of the converted signal by manually changing the input polarization state).

We found that $40 \mathrm{~Gb} / \mathrm{s}$ single polarization conversion shows a moderate power penalty at $10^{-9}$ BER $(1.8 \mathrm{~dB}$ for OOK and 1.3 for DPSK) without any floor tendency. However, PolMux signals suffer from some apparent distortion that always leads to BER floor. These distortions are stronger for the OOK format that has a floor at $10^{-7}$ BER. DPSK performs better and reaches 

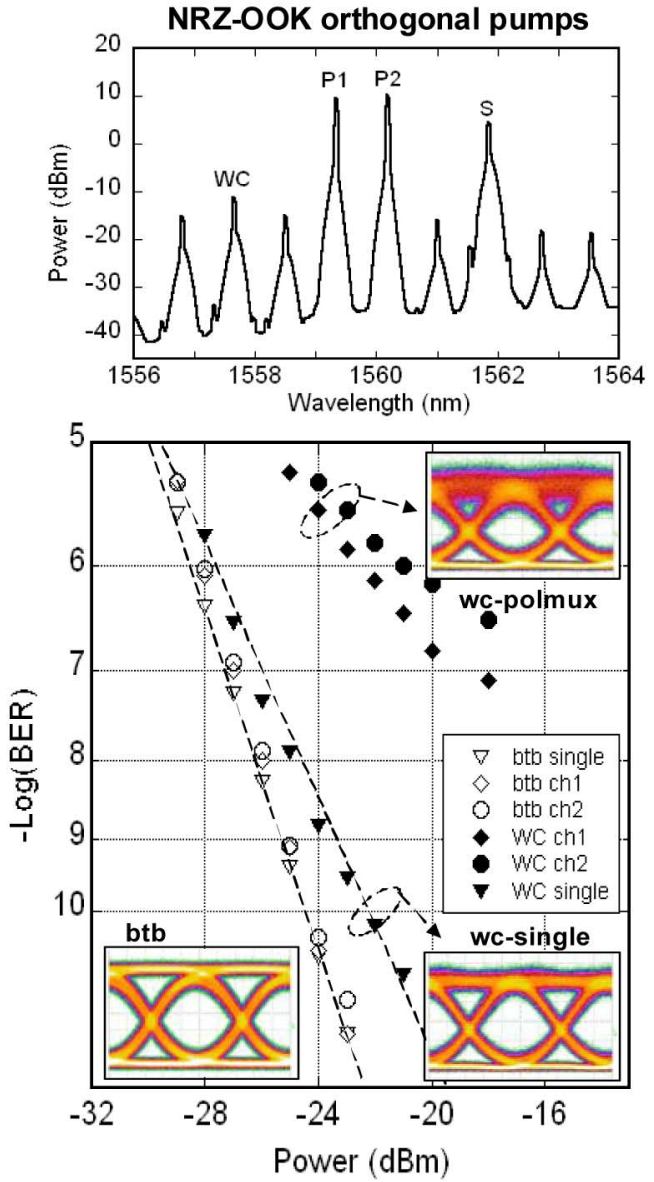

Fig. 3. Optical spectra at the SOA output and BER results for the orthogonal pumps' FWM configuration: 80 and $40 \mathrm{~Gb} / \mathrm{s}$ OOK conversions. Corresponding eye diagrams are shown in the insets.

$10^{-9}$ although with more than $4 \mathrm{~dB}$ power penalty compared to the single polarization conversion. Moreover, in both cases, one of the multiplexed channels performs better than the other one, and this behavior depends on the input polarization state of S, i.e., changing the polarization of S, the BER curves of the multiplexed channels change their relative positions.

\section{FWM With PARAllel PUMPS}

The scheme for parallel pumps is sketched in Fig. 5. In this case, provided that the pumps and signal $\mathrm{S}$ are at wavelengths far enough to neglect their interaction, the nonlinear beating between the two pumps at $\Delta \lambda$ causes the generation of two aside copies (without phase conjugation) of the input signal at a detuning $\pm \Delta \lambda$ and with the same signal polarization state [4]. If the SOA is polarization independent, this process has a constant efficiency for any input polarization. From this, it is evident that the residual polarization dependence of the scheme is at least as large as the PDG of the amplifier.

In this scheme, the conversion detuning is determined by the pump detuning; hence, typically very large conversions are not possible. Nevertheless the scheme has been exploited in a number of applications [8], [9].

We used for the experiment the same setup as in Fig. 2 exploiting a $3 \mathrm{~dB}$ coupler rather than a PBS to couple the pumps.
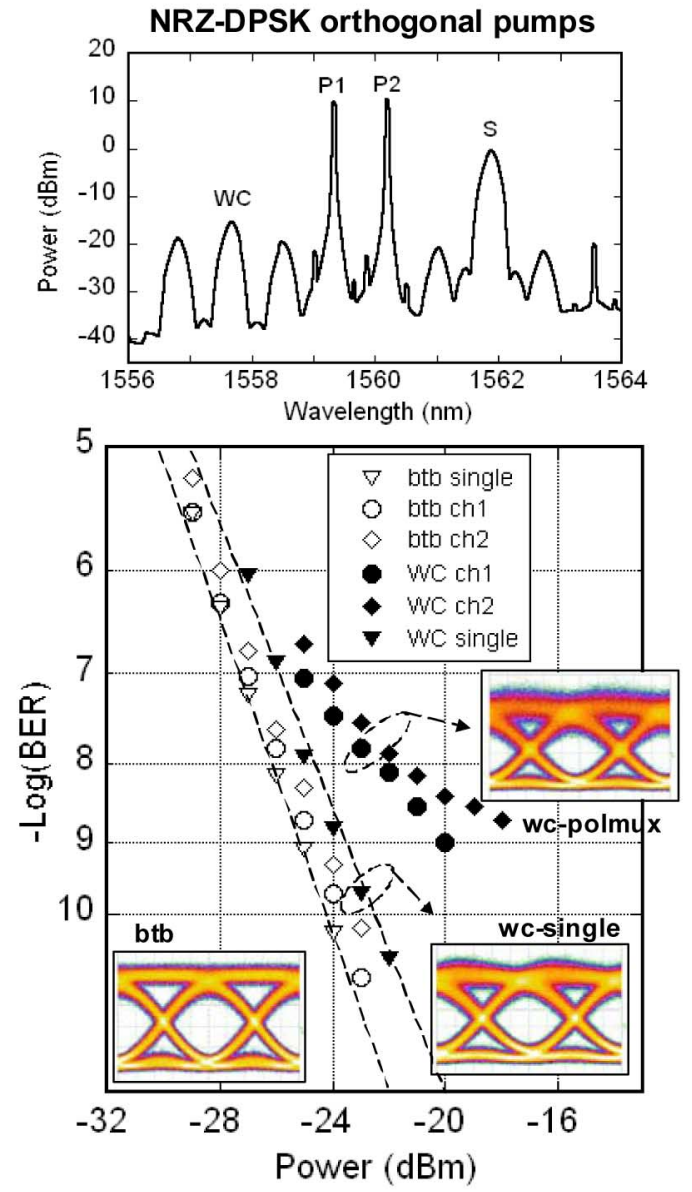

Fig. 4. Optical spectra at the SOA output and BER results for the orthogonal pumps' FWM configuration: 80 and $40 \mathrm{~Gb} / \mathrm{s}$ DPSK conversions. Corresponding eye diagrams are shown in the insets.

Parallel Pumps

(no phase conjugation)

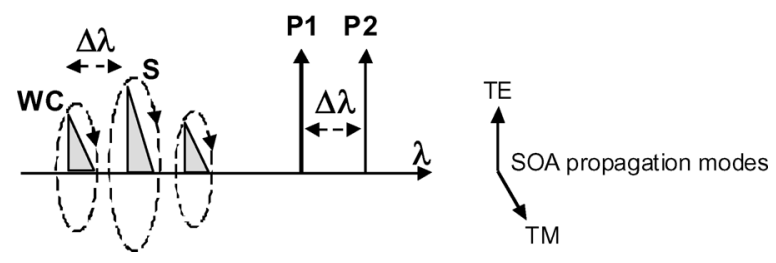

Fig. 5. Schematic of the parallel pumps' FWM configuration.

We optimized the pumps' parallelism by maximizing their FWM products. This corresponds to setting P1 and P2 on the TE or TM axis. We also kept the signal wavelength far enough to avoid the direct FWM interaction with the pumps. All the experimental data are summarized in Tables III and IV. Also, in this case, we choose power levels and wavelength allocation of the signal that gave the best conversion efficiency and output OSNR. All the BER results and the spectra as in the previous case are reported in Fig. 6 for the OOK case and in Fig. 7 for the DPSK case. As we can see from the spectra, in this case, the signal $\mathrm{S}$ is converted into two wavelengths apart from the signal itself. Among them, we selected for the measurements the one that was with the highest power on the shorter wavelength side. The conversion detuning is $0.8 \mathrm{~nm}$, the efficiencies are -10 and 
TABLE III

EXPERIMENTAL POWER LEVELS

\begin{tabular}{|c|c|c|c|c|}
\hline & P1 & P2 & S & WC \\
\hline$\lambda(\mathbf{n m})$ & 1559.6 & 1560.4 & 1553.8 & 1553 \\
\hline $\begin{array}{c}\text { OOK } \\
\text { Power } \\
\text { (dBm) }\end{array}$ & 10.5 & 6.5 & 2.5 & -7.5 \\
\hline $\begin{array}{c}\text { DPSK } \\
\text { Power } \\
\text { (dBm) }\end{array}$ & 11 & 7 & 4 & -7.5 \\
\hline
\end{tabular}

TABLE IV

WAVELENGTH CONVERSION RESULTS

\begin{tabular}{|c|c|c|}
\cline { 2 - 3 } \multicolumn{1}{c|}{} & OOK & \multicolumn{1}{c|}{ DPSK } \\
\hline$\Delta \lambda(\mathbf{n m})$ & 0.8 & 4.2 \\
\hline $\begin{array}{c}\text { Conversion } \\
\text { Efficiency } \\
(\mathbf{d B})\end{array}$ & -10 & -11.5 \\
\hline $\begin{array}{c}\text { OSNR } \\
(\mathbf{d B} / \mathbf{0 . 1 n m})\end{array}$ & 33 & 32 \\
\hline $\begin{array}{c}\text { Residual } \\
\text { pol. depen. } \\
\text { (dB) }\end{array}$ & 1.1 & 1 \\
\hline
\end{tabular}
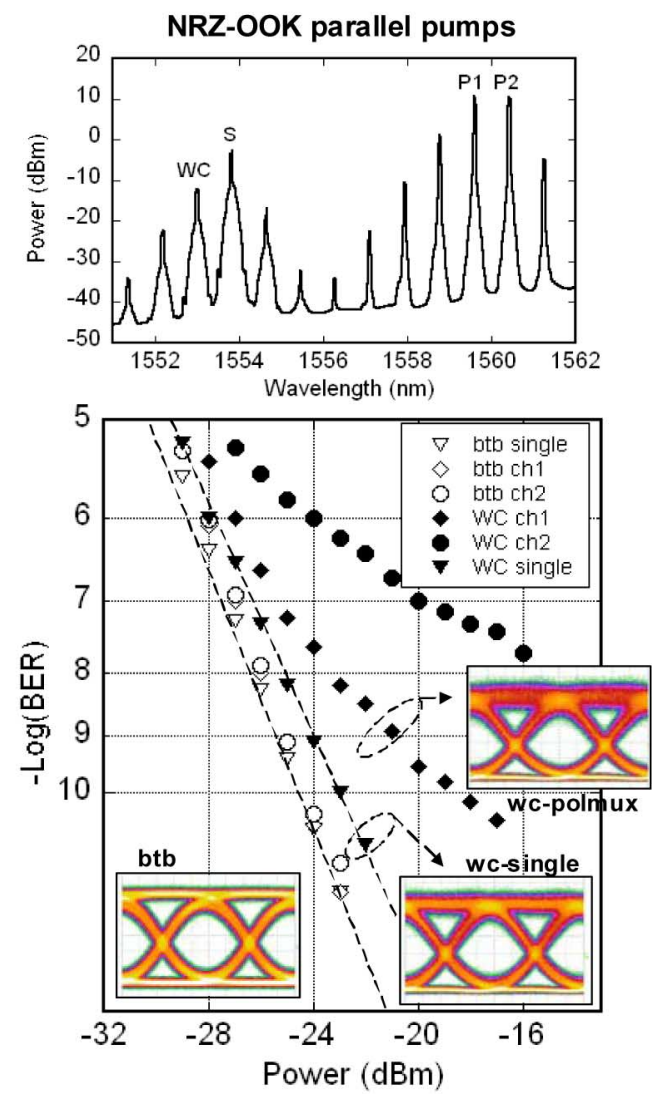

Fig. 6. Optical spectra at the SOA output and BER results for the parallel pumps' FWM configuration: 80 and $40 \mathrm{~Gb} / \mathrm{s}$ OOK conversions. Corresponding eye diagrams are shown in the insets.

$-11.5 \mathrm{~dB}$, and the residual polarization dependence is around $1 \mathrm{~dB}$ (coming mainly from the SOA PDG). Here again, the single polarization conversion performs well, having around
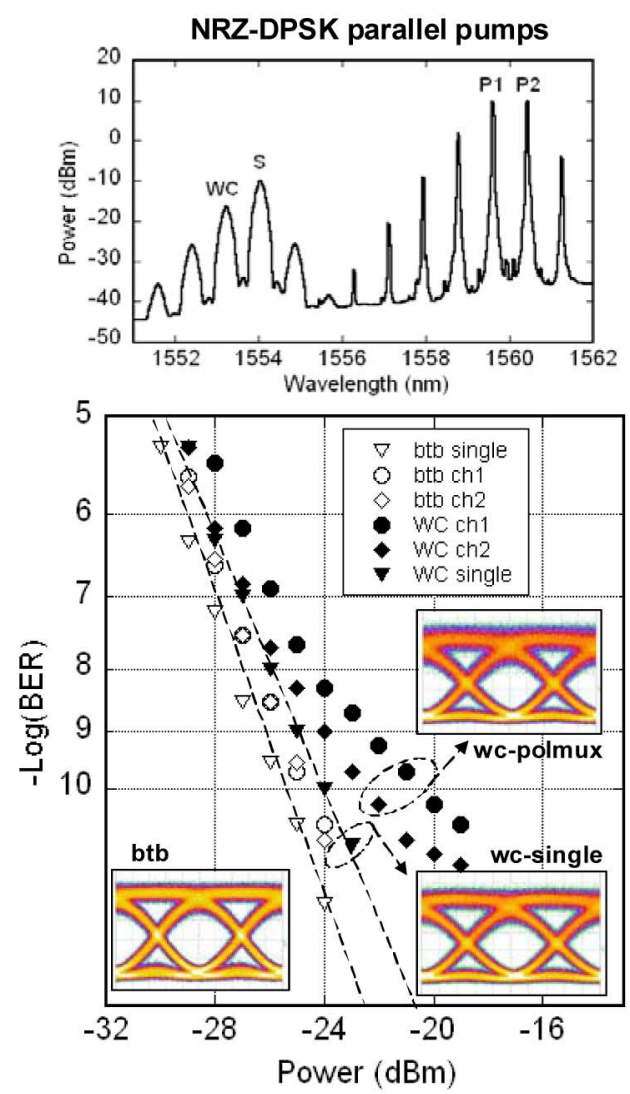

Fig. 7. Optical spectra at the SOA output and BER results for the parallel pumps' FWM configuration: 80 and $40 \mathrm{~Gb} / \mathrm{s}$ DPSK conversions. Corresponding eye diagrams are shown in the insets.

$1.2 \mathrm{~dB}$ power penalty for both OOK and DPSK. DPSK-PolMux has additional penalty, 1.5 and $3 \mathrm{~dB}$, respectively, for the two PolMuxed channels. OOK-PolMux performs worst with one channel suffering from an additional $3 \mathrm{~dB}$ penalty and the other one having a $10^{-8} \mathrm{BER}$ floor. The eye diagrams in the insets give visual information on the signal quality after and before conversion.

\section{DISCUSSION}

We found from the experiments that despite the two schemes we reported here have similar conversion numbers in terms of efficiency, resulting OSNR, and residual polarization dependence, the parallel pumps scheme performs better in all cases. This is probably due to the different inherent working principles that are under the two schemes. However, despite one scheme outperforming the other, still in the best case, the PolMux signals suffer from degradations that could make impractical the cascade of several conversions, especially for OOK signals. We ascribe this degradation to major effects arising from the interplay between the residual polarization dependency of the conversion processes and the nonlinear crosstalk among the PolMuxed channels. First, polarization crosstalk arises from this residual dependence: the wavelength converter acts as a polarization-dependent element that alters the ideal orthogonal condition between the polarization-multiplexed channels. This leads to polarization mixing and therefore to nonlinear interaction between the two polarizations in the SOA. This effect can 


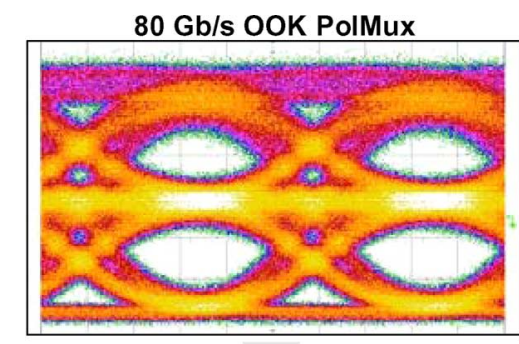

WC

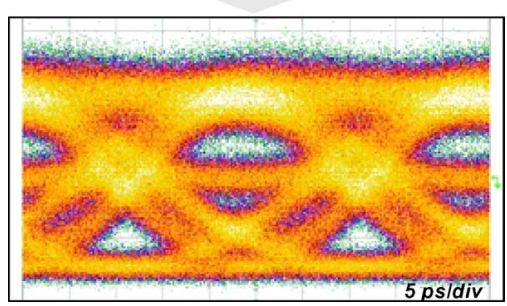

Fig. 8. Example of PolMux eye diagram distortion after wavelength conversion using the orthogonal polarization scheme (before polarization demultiplexing).

also be seen in a different way: as the input PolMux signal is made of two orthogonal decorrelated channels (with no phase coherence), it has a resulting rotating polarization state (at the characteristic frequency of the signal laser linewidth). This polarization rotation is converted into intensity noise by the device dependency to polarization.

Moreover, in both cases, OOK performs even worst because of some small pattern effects that give rise to cross-gain modulation between the two copropagating channels on the orthogonal polarizations in the SOA and those that also interact with previous effects. Because of the constant envelope format, DPSK is less affected by this additional impairment [10].

Waveform distortions can be clearly identified looking at a typical eye diagram of the PolMuxed signals before and after the conversion (before the polarization demultiplexing in the PBS), as reported in Fig. 8. The effects of polarization crosstalk and increased intensity noise are evident in case of $80 \mathrm{~Gb} / \mathrm{s} \mathrm{OOK}$ converted using the orthogonal polarization configuration.

Summarizing, despite the selection of the best conversion parameters in terms of input powers and wavelength detuning and using the best SOA that is commercially available for nonlinear applications, we found that around $1 \mathrm{~dB}$ residual polarization dependence in the conversion process (which is a typical value reported in the literature [4], [6], [8]) together with nonlinear interaction in the SOA is a detrimental factor for a transparent conversion of PolMux signals by using FWM. On the other hand, similar critical issues, which lead to very poor conversion quality, have been found also by other groups performing alternative experiments exploiting coherent techniques for the conversion of PolMux signals [11], [12]. The authors in [11] used FWM in fibers for wavelength conversion of $112 \mathrm{~Gb} / \mathrm{s}$ PolMux RZ-QPSK signals but they found a significant power penalty at very low BER values. Even having only $0.5 \mathrm{~dB}$ residual polarization dependence using a periodically poled $\mathrm{LiNbO}_{3}$ converter in polarization diversity scheme, the authors in [12] found severe conversion penalties. All these results suggest that the demonstration of robust wavelength conversion for PolMux data could be harder than expected.
A possible solution to overcome the impairments could be the use of a polarization diversity scheme with two independent circuits performing FWM in SOAs, as in [13], in which the residual polarization dependence is made the lowest possible (lower than $0.5 \mathrm{~dB}$ ) and PBSs with very large polarization ER are used. In this way, it may be possible to emulate two single-polarization parallel-independent conversions in the two SOAs and to reduce the impairments arising from the polarization splitter/combiner in the PBSs.

\section{CONCLUSION}

We investigated the interplay of advanced modulation formats and all-optical processing that would meet their potential advantages in future optical networks. We experimentally assessed the transparency of FWM in SOAs to phase modulation and PolMux. We used two schemes, which are, in principle, polarization independent, to convert $40 \mathrm{~Gb} / \mathrm{s}$ single polarization and $80 \mathrm{~Gb} / \mathrm{s}$ PolMux signals that were either OOKor DPSK-modulated. We found that although the conversion process works transparently with formats using intensity and phase, transparency to PolMux is a very serious issue. Even just $1 \mathrm{~dB}$ residual polarization dependence can interplay with other nonlinear effects in the conversion and be detrimental in case of PolMux, especially for OOK signals. Similar issues have also been found in other schemes employing coherent nonlinear interactions. These results suggest that transparency to polarization-multiplexed formats is a hard technical goal to be reached to realize future transparent all-optical networks.

\section{REFERENCES}

[1] A. H. Gnauck, R. W. Tkach, A. R. Chraplyvy, and T. Li, "High-capacity optical transmission systems," J. Lightw. Technol., vol. 26, no. 9, pp. 1032-1045, May 2008.

[2] A. H. Gnauck, G. Charlet, P. Tran, P. J. Winzer, C. R. Doerr, J. C. Centanni, E. C. Burrows, T. Kawanishi, T. Sakamoto, and K. Higuma, "25.6-Tb/s WDM transmission of polarization-multiplexed RZ-DQPSK signals," J. Lightw. Technol., vol. 26, no. 1, pp. 79-84, Jan. 2008.

[3] M. Galili, B. Huettl, C. Schmidt-Langhorst, A. Gual i Coca, R. Ludwig, and C. Schubert, "320 Gbit/s DQPSK all-optical wavelength conversion using four wave mixing," in Proc. OFC/NFOEC, 2007, pp. 1-3.

[4] J. P. R. Lacey, M. Summerfield, and S. J. Madden, "Tunability of polarization-insensitive wavelength converters based on four-wave mixing in semiconductor optical amplifiers," J. Lightw. Technol., vol. 16, no. 12, pp. 2419-2427, Dec. 1998.

[5] S. L. Jansen, D. van den Borne, B. Spinnler, S. Calabro, H. Suche, P. M. Krummrich, W. Sohler, G.-D. Khoe, and H. de Waardt, "Optical phase conjugation for ultra long-haul phase-shift-keyed transmission," J. Lightw. Technol., vol. 24, no. 1, pp. 54-64, Jan. 2006.

[6] R. Jopson and R. Tench, "Polarisation-independent phase conjugation of lightwave signals," Electron. Lett., vol. 29, pp. 2216-2217, 1993.

[7] G. Contestabile, R. Proietti, N. Calabretta, M. Presi, A. D'Errico, and E. Ciaramella, "Simultaneous demodulation and clock-recovery of 40-Gb/s NRZ-DPSK signals using a multiwavelength Gaussian filter," IEEE Photon. Technol. Lett., vol. 20, no. 10, pp. 791-793, May 2008.

[8] R. Schnabel, U. Hilbk, T. P. Hermes, Meissner, C. Helmolt, K. Magari, F. Raub, W. Pieper, F. J. Westphal, R. Ludwig, L. Kuller, and H. G. Weber, "Polarization insensitive frequency conversion of a 10-channel OFDM signal using four-wave-mixing in a semiconductor laser amplifier," IEEE Photon. Technol. Lett., vol. 6, no. 6, pp. 56-58, Jan. 1994.

[9] C. Porzi, A. Bogoni, L. Poti, and G. Contestabile, "Polarization and wavelength-independent time-division demultiplexing based on copolarized-pumps FWM in an SOA," IEEE Photon. Technol. Lett., vol. 17, no. 3, pp. 633-635, Mar. 2005.

[10] Y. Dong, Z. Li, J. Mo, Y. Wang, and C. Lu, "Cascade of all-optical wavelength conversion for RZ-DPSK format using four wave mixing in SOA," in Proc. OFC, 2004, vol. 1, pp. 23-27. 
[11] J. Yu and M. Huang, "Wavelength conversion of 112 Gbit/s PolMux RZ-QPSK signals based on four-wave mixing in high-nonlinear fiber using digital coherent detection," in Proc. ECOC, Brussels, Belgium, 2008, vol. 1-27, Paper Mo.3.C.5.

[12] V. Pusino, P. Minzioni, I. Cristiani, V. Degiorgio, L. Marazzi, P. Boffi, M. Ferrario, P. Martelli, P. Parolai, A. Righetti, R. Siano, M. Martinelli, C. Langrock, and M. M. Fejer, "Wavelength conversion of real-time $100 \mathrm{~Gb} / \mathrm{s}$ PolMux RZ-DQPSK," OFC/NFOEC, San Diego, CA 2009, Paper OThS3.

[13] G. Contestabile, A. D’Ottavi, F. Martelli, P. Spano, and J. Eckner, "Broad-band polarization-insensitive wavelength conversion at 10 Gb/s," IEEE Photon. Technol. Lett., vol. 14, no. 5, pp. 666-668, May 2002.

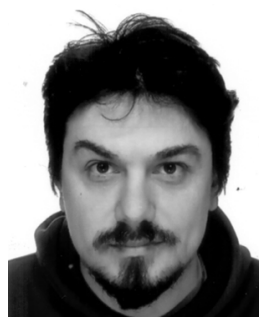

Giampiero Contestabile received the Laurea degree in physics from "La Sapienza" University of Rome, Rome, Italy, in 1998, and the Ph.D. degree in electrical engineering and telecommunications from the "Tor Vergata" University of Rome, Rome, in 2001.

Between 1996 and 2000, he was with the Semiconductor Devices Group, Fondazione Ugo Bordoni, Rome. During 2001, he was with Optospeed Italia. Since September 2002, he has been an Assistant Professor with the Scuola Superiore Sant'Anna, University of Pisa, Pisa, Italy. He has authored or coauthored more than 90 papers published in international peer-reviewed journals and presented in leading international conferences. His current research interests include advanced wavelength-division multiplexing (WDM) systems, optical packet-switched networks, access networks, and applications of semiconductor optical amplifiers. He is one of the reviewers of the JOURNAL OF LightwaVe TeChNOLOGY, Photonics Technology Letters, Optics Express, Optics Letters, and Optics Communications.

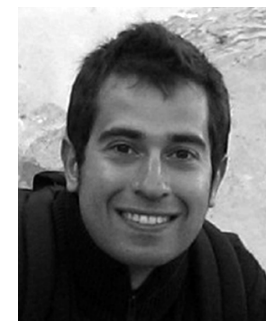

Luca Banchi (S'09) was born in Livorno, Italy, in 1981. He received the Laurea (Master's) degree in telecommunication engineering from the University of Pisa, Pisa, Italy, in 2005. He is currently working toward the Ph.D. degree at the Scuola Superiore Sant'Anna, University of Pisa.

Between September 2005 and December 2007, he was with Elettra Sincrotrone Trieste, where he was involved in the field of FERMI free electron laser. $\mathrm{He}$ is engaged in research on new technical solutions for advanced wavelength-division multiplexing systems.

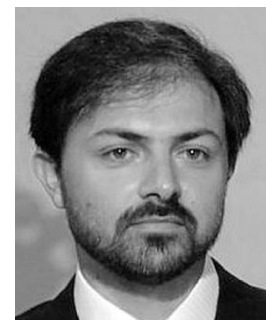

Marco Presi received the Laurea degree in physics from the University of Rome "La Sapienza," Rome, Italy, in 2001, and the Ph.D. degree in applied physics from the University of Pisa, Pisa, Italy, in 2006.

Since 2007, he has been a Research Associate with the Scuola Superiore Sant'Anna, University of Pisa, where he is currently engaged in wavelength-division multiplexing (WDM) optical systems. $\mathrm{He}$ has authored or coauthored more than 50 papers published in international peer-reviewed journals and international conferences.

Ernesto Ciaramella (M'06) was born in Rome, Italy, in 1967. He received the Laurea degree (cum laude) from "La Sapienza" University of Rome, Rome, in 1991.

In 1992, he received a scholarship from Alcatel. During 1992-1994, he was a Researcher with the "Fondazione Ugo Bordoni," where he was engaged in research on nonlinear optical effects, and was a Scientific Researcher during 1998-2000 and was engaged in research on optical transmission systems and network architectures. During 1994-1998, he was with the Centro Studi e Laboratorie Telecommunicazioni S.p.A., Turin, Italy. During 2001-2002, he was a Research Manager at the Consorzio Nazionale Interuniversitario per le Telecomunicazioni (CNIT) National Photonic Networks Laboratory, Pisa, Italy. Since 2002, he has been an Associate Professor with the Scuola Superiore Sant'Anna, University of Pisa, Pisa. His current research interests include optical communications (components, systems, and networks). He participated in various European research projects. He has authored or coauthored approximately 90 papers and five international patents. 